\title{
Zur Kenntnis der peptolytischen Fermente verschiedenartiger Krebse.
}

\author{
Von \\ Emil Abderhalden und Peter Rona.
}

\begin{abstract}
(Aus dem physiologischen Institute der tierärztlichen Hochschule, Berlin, und der chemischen Abteilung des Urban-Krankenhauses, Berlin.)

(Der Redaktion zugegangen am 27. Mai 1909.)
\end{abstract}

Die Fermente der Carcinome waren schon wiederholt Gegenstand von Untersuchungen. Ganz speziell wurden die proteolytischen Zellfermente auf ihre Wirksamkeit geprüft. Bald wurde ein Unterschied gegenüber dem Verhalten der entsprechenden Fermente normaler Zellen gefunden, bald nicht. Die neueste Arbeit stammt von Leo Hess und Paul Saxl.1) Diese Autoren kommen zum Schluß, daß die Carcinomzelle in ihren proteolytisch-fermentativen Eigenschaften vollkommen den Zellen der normalen Gewebe gleicht. Wir sind seit längerer Zeit mit Studien über den Gehalt von Carcinomzellen an peptolytischen Fermenten beschäftigt. Wir haben zunächst festgestellt, wie aus vollständig blutfreiem Carcinomgewebe hergestellter Preßsaft sich gegenüber einem Dipeptid, dem Glycyll-tyrosin, verhält. Es zeigte sich, daß diejenigen Carcinome, welche zu der Gruppe der Adenocarcinome gehören, das genannte Dipeptid genau ebenso spalten, wie normale Gewebszellen. Nur dann, wenn der Krebs ganz aus bindegewebigem Stroma bestand (Scirrhus), fand keine Spaltung des Glycyl-

1) Leo Hess und Paul Saxl, Zur Kenntnis der spezifischen Eigenschaften der Carcinomzelle. 1. Die proteolytischen Fermente. Beiträge zur Carcinomforschung. Heft 1. Urban u. Schwarzenberg. Berlin und Wien. 1909.

Hoppe-Seyler's Zeitschrift f. physiol. Chemie. LX. 
1-tyrosins statt. Unsere Befunde stehen somit, soweit eine Vergleichung zulässig ist, in Einklang mit denen von Hess und Saxl. Die Zahl unserer Versuche ist allerdings noch klein, auch müssen sie nach anderen Richtungen ergänzt werden. Vor allen Dingen muß fetgestellt werden, ob die peptolytischen Fermente der Carcinomzellen sich gegenüber komplizierter gebauten, optisch aktiven Polypeptiden gleich verhalten, wie die entsprechenden Fermente normaler Gewebe. Derartige Versuche sind im Gange.

Die Versuche sind im einzelnen, wie folgt, ausgeführt worden. Es kam nur ganz frisches, eben exstirpiertes Material zur Verwendung. Die Geschwulst wurde sorgfältig von anhaftendem normalem Gewebe und vor allem gründlich von Blut befreit, dann zerkleinert, mit Quarzsand zerrieben, der Brei mit Kieselgur ${ }^{1}$ ) gemischt und durchgeknetet, bis eine plastische Masse entstand. Diese wurde in der oft an dieser Stelle erwähnten Weise bei 300 Atmosphären Druck ausgepreßt. Von dem so erhaltenen Preßsaft wurden jeweilen $10 \mathrm{ccm}$ zu 0,5-0,75 g Glycyl-ltyrosin zugesetzt. Nach Zusatz von einigen Tropfen Toluol blieb die Lösung drei Tage bei $37^{\circ}$ stehen. Die weitere Verarbeitung war die übliche. Die Spaltung des Dipeptids wurde durch die Isolierung des Tyrosins und des Glykokolls festgestellt. Sie ließ sich am ausfallenden Tyrosin leicht verfolgen. Erfolgte keine Abscheidung von Tyrosin, so wurde trotzdem die Lösung auf Spaltprodukte verarbeitet und das ungespaltene Dipeptid als Esterchlorhydrat wiedergewonnen.

1. 16. Februar 1908. Adenocarcinom aus Brustdrüse. $0,5 \mathrm{~g}$ Glycyl-l-tyrosin. Isoliert: 0,16 $\mathrm{g}$ Glykokoll und 0,30 $\mathrm{g}$ Tyrosin.

2. 3. Februar 1908. Scirrhus aus Brustdrüse. 0,5 g Glycyl-l-tyrosin. Isoliert: 0,05 g Glykokoll und 0,10 g Tyrosin. Ferner 0,25 g Glycyl-l-tyrosin.

3. 18. Februar 1908. Adenocarcinom aus Brustdrüse. $0,75 \mathrm{~g}$ Glycyl-l-tyrosin. Isoliert: $0,18 \mathrm{~g}$ Glykokoll und $0,40 \mathrm{~g}$ Tyrosin.

1) Nach neueren Erfahrungen wird der Zusatz von Kieselgur besser vermieden. 
4. 15. Januar 1908. Adenocarcinom aus Brustdrüse. 0,5 g Glycyl-l-tyrosin. Isoliert: $0,12 \mathrm{~g}$ Glykokoll und 0,30 $\mathrm{g}$ Tyrosin.

5. 21. Januar 1908. Adenocarcinom aus Brustdrüse. 0,5 g Glycyl-l-tyrosin. Isoliert: $0,08 \mathrm{~g}$ Glykokoll und 0,20 $\mathrm{g}$ Tyrosin.

6. 18. Februar 1908. A denocarcinom aus Brustdrüse. 0,5 g Glycyl-l-tyrosin. Isoliert: $0,14 \mathrm{~g}$ Glykokoll und 0,32 $\mathrm{g}$ Tyrosin.

7. 24. Februar 1908. Scirrhus aus Brustdrüse. 0,5 g Glycyl-l-tyrosin. Isoliert: $0 \mathrm{~g}$ Glykokoll, $0 \mathrm{~g}$ Tyrosin und $0,42 \mathrm{~g}$ Glycyl-l-tyrosin.

8. 26. Februar 1908. Adenocarcinom aus Brustdrüse. $0,5 \mathrm{~g}$ Glycyl-l-tyrosin. Isoliert: $0,10 \mathrm{~g}$ Glykokoll und $0,25 \mathrm{~g}$ Tyrosin.

9. 5. Mai 1908. Scirrhus aus Brustdrüse. 0,5 g Glycyl1-tyrosin. Isoliert: $0,05 \mathrm{~g}$ Glykokoll und $0,10 \mathrm{~g}$ Tyrosin, ferner $0,32 \mathrm{~g}$ Glycyl-l-tyrosin.

10. 3. Mai 1908. Scirrhus aus Brustdrüse. $0,5 \mathrm{~g}$ Glycyll-tyrosin. Isoliert: $0,39 \mathrm{~g}$ Glycyl-l-tyrosin. Keine Spaltprodukte aufgefunden.

11. 22. Dezember 1907. Adenocarcinom aus Brustdrüse. $0,5 \mathrm{~g}$ Glycyl-l-tyrosin. Isoliert: $0,13 \mathrm{~g}$ Glykokoll und 0,32 g Tyrosin.

12. 18. Februar 1908. Myoma uteri. $0,75 \mathrm{~g}$ Glycyll-tyrosin. Isoliert: $0,10 \mathrm{~g}$ Glykokoll und 0,28 $\mathrm{g}$ Tyrosin, ferner $0,25 \mathrm{~g}$ Glycyl-l-tyrosin. 